bioRxiv preprint doi: https://doi.org/10.1101/348326; this version posted June 16, 2018. The copyright holder for this preprint (which was not

\title{
Frequent translation of small open reading frames in evolutionary conserved IncRNA regions
}

Jorge Ruiz-Orera ${ }^{1, *}$ and M.Mar Albà ${ }^{1,2, *}$

${ }^{1}$ Evolutionary Genomics Group, Research Programme on Biomedical Informatics, Hospital del Mar Research Institute, Universitat Pompeu Fabra, Barcelona, Spain; ${ }^{2}$ Catalan Institution for Research and Advanced Studies, Barcelona, Spain.

*To whom correspondence should be addressed.

Keywords: ribosome profiling, translation, RNA-protein interaction, long non-coding RNA, evolution 


\section{SUMMARY}

The mammalian transcriptome includes thousands of transcripts that do not correspond to annotated protein-coding genes. Although many of these transcripts show homology between human and mouse, only a small proportion of them have been functionally characterized. Here we use ribosome profiling data to identify translated open reading frames, as well as nonribosomal protein-RNA interactions, in evolutionary conserved and non-conserved transcripts. We find that conserved regions are subject to significant evolutionary constraints and are enriched in translated open reading frames, as well as non-ribosomal protein-RNA interaction signatures, when compared to non-conserved regions. Translated ORFs can be divided in two classes, those encoding functional micropeptides and those that show no evidence of protein functionality. This study underscores the importance of combining evolutionary and biochemical measurements to advance in a more complete understanding of the transcriptome. 


\section{INTRODUCTION}

2 The advent of high-throughput genomic technologies has revealed that mammalian transcrip-

3 tomes are more complex than initially thought (Carninci et al., 2005; Kapranov et al., 2007;

4 Okazaki et al., 2002; Ponjavic et al., 2007). One of the most intriguing findings has been the dis-

5 covery of thousands of expressed loci that lack conserved or long ORFs (Cabili et al., 2011; Liu

6 et al., 2012; Okazaki et al., 2002; Pauli et al., 2012; Ponting et al., 2009; Ulitsky and Bartel,

7 2013). These transcripts, commonly denominated long non-coding RNAs (IncRNAs), share

8 many of the features of coding mRNAs, such as the presence of a polyadenylated tail and a

9 multi-exonic structure (Consortium et al., 2007). Some IncRNAs may have originated as a result

10 of bidirectional transcription from promoters (Lepoivre et al., 2013) or enhancers (Hon et al.,

11 2017; Li et al., 2016), whereas others may have been born thanks to the fortuitous appearance

12 of weak promoters in the genome (Ruiz-Orera et al., 2015).

13 The function of IncRNAs is a matter of intense debate. In general, IncRNAs display high evolu-

14 tionary turnover (Kutter et al., 2012; Neme and Tautz, 2016), and show very weak sequence

15 constraints according to single nucleotide polymorphism data (Wiberg et al., 2015). This is con-

16 sistent with the idea that many IncRNAs are not functional but a result of the high transcriptional

17 activity of the genome (Brosius, 2005; Struhl, 2007; Wang et al., 2004). However, some IncRNAs

18 have been shown to regulate gene expression through interactions with specific proteins in the

19 nucleus or the cytoplasm (Gong et al., 2012; Han et al., 2010; Ribeiro et al., 2017) or with the

20 chromatin (Ponting et al., 2009), even if expressed at very low levels (Seiler et al., 2017). Cur-

21 rently, the fraction of IncRNAs that are functional is unknown.

22 Not surprisingly, functional IncRNAs often contain short conserved sequence segments that are

23 required for their function (Kapusta and Feschotte, 2014; Ulitsky, 2016). Although very few IncR-

24 NAs display deep conservation in vertebrates, hundreds of IncRNAs show conservation between

25 human and mouse (Necsulea et al., 2014; Ulitsky et al., 2011). The conserved sequence 
patches tend to be short and 5'-biased (Hezroni et al., 2015). According to polymorphism data,

27 the evolution of conserved IncRNAs tends to be more constrained than the evolution of non-con-

28 served IncRNAs (Wiberg et al., 2015), indicating that the former IncRNAs are enriched in func-

29 tional sequences.

30 Ribosome profiling sequencing data (Ribo-Seq), which captures RNA-ribosome interactions but

31 also other types of RNA-protein interactions (Ingolia et al., 2009; Ji et al., 2016), offers new op-

32 portunities to investigate the properties of conserved versus non-conserved IncRNA regions.

33 Remarkably, Ribo-Seq has revealed the existence of thousands of translated open reading

34 frames (ORFs) in IncRNAs (Bazzini et al., 2014; Castaneda et al., 2014; Ingolia et al., 2011; Ji et

35 al., 2015; Ruiz-Orera et al., 2014). Some of them correspond to small functional proteins which

36 have been missed by gene annotation pipelines, such as myoregulin (Mrln) (Anderson et al.,

37 2015) or TUNAR (Megamind) (Lin et al., 2014). Other ORFs are likely to translate non-functional

38 peptides according to polymorphism data (Ruiz-Orera et al., 2014, 2018). The footprints of non-

39 ribosomal ribonucleoprotein particles have also been detected on some functional IncRNAs be-

40 cause of the distinctive length of the associated Ribo-Seq reads (Ingolia et al., 2014).

Here we investigate the presence of Ribo-Seq-related signatures, as well as other annotated

43 features such as putative promoter sequences, in IncRNAs sequences that are conserved

44 between mouse and human. We find that conserved regions contain an excess of promoter

45 sequences, translated ORFs and non-ribosomal ribonucleoprotein particles when compared to 46 non-conserved regions.

47

\section{RESULTS}


52 We searched for matches of the complete set of Ensembl mouse annotated transcripts against

53 the human transcriptome using BLASTN (E-value $<10^{-5}$ ) (Altschul et al., 1997). The human

54 transcriptome was obtained using high coverage RNA sequencing data (RNA-Seq) from different

55 tissues (Ruiz-Orera et al., 2015). We detected 19,779 conserved protein-coding genes

56 (codRNAs) and 1,547 conserved IncRNAs, containing at least one conserved region in humans.

57 The conserved regions were in general shorter in IncRNAs than in codRNAs (median length of

58163 and 343 nucleotides, respectively; Additional file 1: Figure S1 for more details).

60 Next, we focused on genes expressed that were significantly expressed in brain tissue, using a

61 high coverage ribosome profiling dataset from mouse hippocampus (Cho et al., 2015). We

62 detected significant expression for 13,081 conserved codRNAs and 289 conserved IncRNAs,

63 including 444 conserved IncRNA regions (Additional file 1: Table S1). The set of conserved

64 IncRNAs was enriched in functionally characterized IncRNAs (27 cases, see Additional File 2);

65 the only exceptions were Firre, Adapt33, and Snhg6. The percentage of genes with at least one

66 conserved region was $98 \%$ for codRNAs and $40.88 \%$ for IncRNAs (Figure 1a). In terms of total

67 length, $8.50 \%$ of the total IncRNA sequence $(25.39 \%$ in the case of functionally characterized

68 IncRNAs), and $61.62 \%$ of the codRNA sequence, was conserved (Figure 1a).

69

70 We observed that the transcripts frequently overlapped sequences annotated as promoters by

71 Ensembl (Zerbino et al., 2015). This affected $87.15 \%$ of codRNAs and $68.18 \%$ of IncRNAs. In

72 relative terms, IncRNAs were more extensively covered by promoters $(24.50 \%$ of total

73 sequence) than codRNAs (11.52\% of total sequence) (Figure 1a), and promoter regions were

74 biased towards the $5^{\prime}$ end of IncRNAs (Figure $1 b$ ). The relatively high overlap of promoters with

75 IncRNAs could be explained by their short size compared to codRNAs; when we focused on the

765 '-most 200 nucleotides of the transcript the percentage of sequence covered by promoters was

77 actually higher for codRNAs than for IncRNAs (80.46\% versus $63.38 \%$ ). We observed a strong

78 enrichment of promoters in conserved regions: promoters occupied nearly $54 \%$ of the total

79 IncRNA conserved sequence, compared to only $\sim 22 \%$ of the non-conserved one (Figure $2 b$ ). 
81 Next, we investigated the presence of ribosome profiling (Ribo-Seq) signals on the transcripts.

82 We observed that most codRNAs (99.10\%) and IncRNAs (89.92\%) were covered by at least 1

83 Ribo-Seq read. When looking at the total sequence length, 50.08\% of the codRNA sequence

84 and $27.17 \%$ of the IncRNA sequence was covered by Ribo-Seq reads (Figure 1a). These results

85 are in line with recent reports of a relatively high coverage of IncRNAs by Ribo-Seq reads

86 (Ingolia et al., 2011; Ruiz-Orera et al., 2014). The Ribo-Seq reads showed a clear 5' bias, both in

87 conserved and non-conserved regions (Figure 1b, Additional file 1: Figure S2).

89 To account for the fact that some regions might be conserved because of antisense overlaps

90 with protein-coding exons, we did the same analysis without considering any overlapping region,

91 reaching similar conclusions of higher density of Ribo-Seq reads in conserved versus non-

92 conserved regions (Additional file 1: Figure S3). Moreover, even though conserved regions had

93 higher RNA-seq and Ribo-Seq read coverage than non-conserved ones (Additional file 1: Figure

94 S4), the same effect could be observed for different expression level intervals, indicating that the

95 trend was robust to variations in the amount of the transcript (Additional file 1: Figure S5). Ribo-

96 Seq data from human and rat brain tissues, for the corresponding genomic syntenic sequences,

97 yielded very similar results (Additional file 1: Figure S6).

98

Consistent results across IncRNA types

100

101 We divided IncRNAs come in two groups, intergenic IncRNAs (I, Figure 2a) and antisense

102 IncRNAs (A, figure 2a). Intergenic IncRNAs were completely independently loci. Antisense

103 IncRNAs included those IncRNAs annotated as antisense in Ensembl, as well as any other

104 IncRNA whose transcription start site was located less than $2 \mathrm{~Kb}$ from the TSS of another gene in

105 antisense orientation and/or had antisense exonic overlap with another gene. We also found 50

106 annotated IncRNAs with embedded short non-coding RNAs in the exons (they contain 33

107 miRNAs, 41 snoRNAs, and 32 miscRNAs); we termed this class ncRNA host (H, Figure 2a). 
108 Many of these IncRNAs are known to be processed to form small conserved RNA molecules, as

109 it occurs with the 3' tail of Malat (Wilusz et al., 2008), although in other cases (f.e. Slert) the

110 presence of the SRNA-like sequence in the IncRNA enables the biogenesis and translocation of

111 the transcript (Xing et al., 2017). For comparative purposes, we classified the genes annotated

112 as coding in the same three categories as the IncRNAs. We observed that the relative frequency

113 of antisense genes was much higher in IncRNAs than in codRNAs (451 out of 707 versus 3,324

114 out of 13,342).

115

116 The class defined as ncRNA host was strongly enriched in conserved sequences when 117 compared to the other two IncRNA classes (Figure 2a). Overall, 90\% of the mouse ncRNA host

118 sequences showed significant conservation in the human transcriptome, whereas this fraction 119 was $42 \%$ for antisense IncRNAs and $27 \%$ for intergenic IncRNAs. Promoter sequences and 120 Ribo-Seq mappings were more abundant in conserved than in non-conserved regions for all 121 three IncRNA classes (Figure 2b). The main differences between the classes were an excess of 122 promoter sequences in antisense IncRNAs and increased Ribo-Seq signal in conserved ncRNA 123 host. When the three classes of IncRNAs were taken together, the fraction of regions covered by 124 Ribo-Seq reads was about double for conserved than for non-conserved regions (51.7\% versus $12524.9 \%$, Test of equal proportions, p-value $\left.<10^{-5}\right)$.

\section{Conserved IncRNA sequences are under selection}

129 Although mouse and human are relatively distant species ( $~ 90$ Million years) (Hedges et al., 130 2015), some sequence segments may be conserved purely by chance. In order to estimate the 131 expected degree of conservation between mouse and human transcripts in the absence of 132 selection we run sequence evolution simulations using Rose (Stoye et al., 1998). In particular, 133 we simulated the evolution of IncRNAs along the mouse and human branches under no 134 evolutionary constraints. Subsequently we performed BLASTN searches of the evolved mouse 135 sequences against the set of evolved human sequences (see Methods for more details). We 
136 could find BLASTN homology hits for about $56.2 \%$ of the evolved IncRNA sequences. The fact

137 that this fraction is larger than the observed one for real IncRNAs (40.9\%, Figure 1a) supports

138 the idea that a fraction of the current mouse IncRNAs have originated after the split with the

139 human lineage.

140

141 Next, we used the sequence alignments obtained with BLASTN to estimate the number of

142 substitutions per site ( $k$ ) using the PAML package (Yang, 2007), in different sequence sets. In

143 alignments of size equal or longer than 300 nucleotides, the computed $k$ was similar to the

144 expected 0.51 substitutions per site for regions evolving under no constraints (See Methods for

145 more details). Using the same length cut-off the computed $k$ for real IncRNAs was 0.25 and

146 hence significantly lower than the expected under no constraints (Wilcoxon test, $p$-value $<10^{-5}$ ).

147 This indicates that purifying selection is acting on IncRNAs containing regions that are

148 conserved between mouse and human.

150 Alignments shorter than 300 nucleotides tended to give estimates of $k$ lower than 0.51 even in

151 the case of the simulated sequences, which was not initially expected. In this size range we

152 observed a positive relationship of $k$ with alignment length, with shorter sequences showing

153 lower $k$ (Additional file 1: Figure S7). This indicated that short sequenced needed to have a

154 higher percent identity to be detected as significant by BLAST. As many conserved sequences in

155 IncRNAs were lower than 300 nucleotides (Additional file 1: Figure S1) we modeled the effect of

156 length on $k$ using two different log-linear regression models, one for short $(<300 \mathrm{nt})$ and one for

157 long ( $\geq 300 \mathrm{nt})$ sequences, using the data from the sequence evolution simulations. This allowed

158 to predict an expected $k\left(k_{\underline{e}}\right)$ given a sequence alignment length, which we used to normalized

159 the observed $k$ for real sequences $\left(k_{0} / k_{e}\right)$.

161 The $k_{o} / k_{e}$ was significantly lower in all three categories of IncRNAs than in the neutrally evolved

162 sequences (Figure 3, Wilcoxon test, $\mathrm{p}$-value $<10^{-5}$ ), consistent with selection acting on at least

163 some of the IncRNAs. The intensity of the selection signal, as measured with the $k_{o} / k_{e}$ ratio, was 
164 similar for conserved segments in functionally characterized and uncharacterized IncRNAs

165 (median 0.49 and 0.50 , respectively). Coding sequences and ncRNA host transcripts also

166 showed clear selection signals (median $k_{o} / k_{e} 0.37$ and 0.46 , respectively). We also calculated

167 separated values for regions corresponding to Ensembl annotated promoter regions, which

168 spanned $34-69 \%$ of the conserved IncRNA regions (Figure 2b). Although conserved promoter

169 regions had a somewhat lower $k_{d} / k_{e}$ than the rest of conserved IncRNA sequences (median 0.47

170 versus 0.58 ), the signal of purifying selection continued to be very clear after eliminating the

171 promoters.

\section{Conserved IncRNAs regions are enriched in translated ORFs}

174

175

Actively translated sequences show a characteristic three-nucleotide read periodicity in ribosome profiling experiments, allowing the identification of novel translation events (Bazzini et al., 2014; Chew et al., 2013; Ingolia et al., 2009). We used the program RibORF to score read periodicity and uniformity in all ORFs of size 30 nucleotides or longer (Ji et al., 2015). Translated ORFs were defined as those with a RibORF score equal or higher than 0.7 , as previously described (Ruiz-Orera et al., 2018) (Figure 4a). The program predicted that $52.05 \%$ of all expressed IncRNAs translated at least one ORF, which is in line with previous studies (Calviello et al., 2016; Ji et al., 2015; Ruiz-Orera et al., 2014, 2015). Annotated functional IncRNAs were no exception; we found significant Ribo-Seq signal in 29 out of 30 annotated functional IncRNAs. Except for TERC, Rian, Mir124a-1hg, and Kcnq1ot1, the rest of the cases contained small ORFs that appeared to be translated (Additional File 2: Table 3). Virtually all codRNAs with conserved regions were translated; in the case of IncRNAs with conserved regions this fraction was $57.1 \%$ (Figure 4b).

Overall, about $14.1 \%$ of the total conserved region in IncRNAs contained ORFs predicted to be translated (122 ORFs), compared to $5.65 \%$ for non-conserved regions (370 ORFs). The enrichment of translated ORFs in conserved regions was highly significant (Figure 4c, Test of 
192 equal proportions, $\mathrm{p}$-value $\left.<\mathbf{1 0}^{-5}\right)$. A similar result was observed after discarding regions

193 overlapping other genes in antisense direction (Additional file 1: Figure S3). We also observed

194 that the translated ORFs were more abundant in the $5^{\prime}$ end than in the 3 ' end of genes,

195 independently of mouse-human sequence conservation (Additional file 1: Figure S4). This may

196 be related to the ribosome scanning dynamics, starting at the $5^{\prime}$ end of transcripts, and perhaps

197 also to the higher GC content in this part of the gene (Additional file 1: Figure S8), which may

198 favor the presence of ORFs (Vakirlis et al., 2018). The enrichment was consistently observed

199 across the different IncRNA subtypes, with translation occurring more actively in antisense

200 genes than in other IncRNA classes (Figure 4c).

202 We next investigated if the putative translated ORFs in IncRNA conserved regions showed 203 signatures of selection at the protein level (Figure 4a). Out of the 93 cases in which we could

204 recover and align the corresponding human sequences using genomic alignments, 10 showed a

205 ratio of non-synonymous to synonymous rates (dN/dS) significantly lower than 1 (chi-square

206 test, $p$-value $<0.05)$, indicating that this subset of translated products might be functional. The

207 size of the new putative proteins ranged from 19 to 128 amino acids and they were located in

208 uncharacterized IncRNAs (Additional File 2: Table 4). Even though many annotated functional

209 IncRNAs had ORFs in conserved regions, none of them had significant signatures of selection at

210 the protein sequence level. For comparison we also analyzed the signatures of selection in 157

211 conserved and 38 not conserved codRNA genes encoding small proteins (small CDSs, $<100$

212 amino acids). In this case a much higher proportion of the aligned cases (76 out of 124) showed

213 significant negative selection signatures. These cases included a number of known functional

214 peptides such as Myoregulin (Anderson et al., 2015; Yu et al., 2017), Tunar (Lin et al., 2014),

215 NoBody (D'Lima et al., 2017), or CASIMO1 (Polycarpou-Schwarz et al., 2018), originally

216 annotated as IncRNAs; and other small functional peptides such as Stannin (Buck-Koehntop et

217 al., 2005; Pueyo et al., 2016), or Sarcolipin (Magny et al., 2013; Wawrzynow et al., 1992). 
219 We analyzed PhyloP scores for $+1,+2$, and +3 positions in codons (see Methods) and we

220 observed that small CDSs had lower conservation values for nucleotides in the third position,

221 which has been generally observed for annotated proteins (Pollard et al., 2010). However, only

222 conserved ORFs with dN/dS-based evidence of negative selection in IncRNAs had the same

223 bias in the third position (Additional file 1: Figure S9). We concluded that, although IncRNA

224 conserved regions are enriched in putatively translated ORFs, probably only a relatively small

225 subset of them are producing functional peptides.

\section{Identification of RNA-protein interactions}

Analysis of fragment length on Ribo-Seq data has revealed differences in the patterns of

230 sequences bound to ribosomes and to small RNAs (Ingolia et al., 2014; Ji et al., 2016). When

231 analyzing the regions covered by Ribo-Seq reads we found that most codRNAs were covered by

232 reads with lengths of 30-32 nucleotides, which correspond to ribosome associations. In IncRNAs

233 the length of the Ribo-Seq reads was more variable, as would be expected if, in addition to

234 translated ORFs, there were non-ribosomal protein-RNA interactions, or ribonucleoproteins

235 (RNPs). The excess of short (<30 nt) and long ( $>32 \mathrm{nt})$ reads could be clearly observed in

236 intergenic IncRNAs and ncRNA host (Figure 5a). Similar patterns of Ribo-Seq read length were

237 observed in another ribosome profiling experiment from rat when looking at the syntenic regions

238 (Additional file 1: Figure S10).

240 We searched for candidate RNP regions by first identifying regions with low Ribo-Seq read 241 uniformity (< 0.6) with the program Rfoot (Ji et al., 2016), and then checking that the Ribo-Seq

242 reads spanning these regions had lengths which were more compatible with RNPs than with 243 ribosome associations using the previously developed FLOSS methodology (Ingolia et al., 244 2014). In particular, we selected RNP candidates with a FLOSS divergence score $\geq 0.35$ (Figure

$2454 a$ and Additional file 1: Figure S11). This procedure identified 134 conserved regions in 84 246 genes that had RNP signatures. This included 21 annotated IncRNAs known to be involved in 
247 different functional protein interactions (Additional File 2: Table 3). Among them there were

248 Malat, Neat1, Meg3, and Miat, known to interact with different protein and splicing factors, and

249 TERC, which acts as a scaffold for the telomerase complex. We also found 32 uncharacterized

250 antisense IncRNAs, 12 intergenic IncRNAs, and 19 ncRNA host genes that showed sequence

251 conservation in humans and were associated with RNPs (Additional File 2: Table 2). RNP

252 regions had normalized substitution rates $\left(k_{0} / k_{e}\right)$ lower than the simulated sequence evolution

253 control (Wilcoxon test, $\mathrm{p}$-value $<10^{-5}$ ), but not different from conserved IncRNA regions in

254 general.

256 There was an enrichment of RNP signaures in transcripts with at least one conserved region

257 when compared to transcripts with no conserved regions (Figure 4b, Conserved versus Not

258 conserved). The trend was highly significant for intergenic IncRNAs, with $15 \%$ of the conserved

259 regions covered by putative RNPs (Figure 4c). Among non-conserved genes with RNP

260 signatures we identified Firre, a functional IncRNA that interacts with nuclear factors through a

261 repetitive sequence (Hacisuleyman et al., 2014). In this IncRNA, the predicted RNPs matched

262 the repetitive sequences.

The RNP signatures were clearly lower in codRNAs than in IncRNAs. For example, whereas in

265 IncRNAs read coverage was similar for RNPs and translated ORFs, in codRNAs the translated

266 ORFs had higher coverage (Figure 5b). In conserved IncRNA regions, RNPs and ORFs

267 occupied a similar percentage of the sequence $(17.2 \%$ and $14.1 \%$, respectively). In contrast, in

268 conserved codRNA regions, RNPs only occupied $1.7 \%$ of the sequence, whereas ORFs

269 occupied 65.5\% (Additional file 1: Figure S12).

\section{DISCUSSION}

273 Here we performed an evolutionary analysis of the mouse transcriptome and studied the 274 relationship between evolutionary conservation and the presence of regulatory elements and 
275 Ribo-Seq-related features. Several previous studies used regions of predefined genomic

276 synteny to identify homologous regions with primary sequence conservation (He et al., 2015;

277 Hezroni et al., 2015; Li and Yang, 2017; Mohammadin et al., 2015; Ulitsky et al., 2011). These

278 studies showed that IncRNAs were less conserved across distant species than protein-coding

279 genes (Guttman et al., 2009; Marques and Ponting, 2009; Necsulea et al., 2014). However,

280 genomic conservation does not always imply conserved IncRNA expression and/or functionality.

281 LncRNAs are known to have a high expression turnover (Kutter et al., 2012; Neme and Tautz,

282 2016; Ruiz-Orera et al., 2015) and thus IncRNA expression is often species-specific or limited to

283 very close species, even when genomic syntenic regions can be identified in more distant

284 species (Hezroni et al., 2015). In order to circumvent these limitations here we focused on

285 sequences expressed both in mouse and human, and which had significant sequence similarity

286 by BLASTN, denoting common ancestry. We identified $289(40.88 \%)$ IncRNAs expressed in

287 hippocampus with homology to human transcripts. Conserved regions in IncRNA were usually

288 small; they occupied $8.50 \%$ of the total mouse IncRNA sequence length. Although these regions

289 were small, we have to consider that a short region may in some cases be sufficient to carry out

290 the function of the IncRNA (Quinn et al., 2014). In some cases, exon structures located in the 3'

291 region may be rewired without necessarily affecting IncRNA and/or promoter function, as it

292 occurs with the Pvt1 gene (Hezroni et al., 2015).

294 Previous studies found that IncRNAs conserved across different species were more constrained

295 than species-specific IncRNAs (Kutter et al., 2012; Wiberg et al., 2015) or that sequences

296 presumably evolving under no constraints (Marques and Ponting, 2009). It was also reported

297 that putative low-accessibility nucleotides from secondary structure elements showed a

298 depletion of polymorphisms when compared to other exonic and intronic sequences (Pegueroles

299 and Gabaldón, 2016). Here we estimated the nucleotide substitution rate ( $k$ ) from mouse and

300 human IncRNA aligned regions, and compared it to the expected one for sequences evolving

301 under no constraints. We found that, in general, conserved regions in IncRNAs had significantly

302 lower substitution rates than neutrally evolved sequences. This finding is consistent with the 
303 existence of evolutionary constraints in IncRNA conserved regions; those positions that are

304 important for the function of the transcript will tend to change less than expected by chance.

305 However, we also have to consider that the mutation rate may be quite heterogeneous in

306 different genomic locations and that this may generate biases that are not related to selection

307 and which are difficult to model.

309 We found that IncRNA conserved regions were frequently located in the $5^{\prime}$ end of transcripts and

310 that they frequently overlapped with putative promoter sequences. This is in line with previous

311 observations that promoters of conserved mammalian IncRNAs tend to show low sequence

312 divergence (Derrien et al., 2012; Guttman et al., 2009). This pattern may be explained by

313 selection acting to maintain the expression of the gene, but there may also be a certain degree

314 of ascertainment bias, as homology searches will favor the detection of transcripts with

315 conserved promoters even if selection is not acting.

317 In many cases, regions other than promoters were conserved and associated with low

318 substitution rates. This included 95\% of the transcripts hosting small RNAs, which are expected

319 to contain functional RNA molecules, but also $27 \%$ of the intergenic and $42 \%$ of the antisense

320 IncRNAs. As it has been previously observed that IncRNAs often contained ribosome profilign

321 signatures (Aspden et al., 2014; Bazzini et al., 2014; Guttman et al., 2013; van Heesch et al.,

322 2014; Ingolia et al., 2011; Juntawong et al., 2014; Ruiz-Orera et al., 2014), we hypothesized that

323 evoutionary conservation could be related to the presence of translated ORFs or non-ribosomal

324 ribonucleoprotein particles in the transcripts. We analyzed the patterns of Ribo-Seq in a mouse

325 hippocampus dataset and observed a Ribo-Seq bias towards the 5' end fraction of both coding

326 and non-coding transcripts. Remarkably, our approach found a very significant enrichment of

327 Ribo-Seq reads in IncRNA conserved regions. The findings were consistent across different 328 expression ranges and species, strengthening our conclusions. 
330 The presence of Ribo-Seq signal in IncRNAs has been previously proposed to be the result of

331 the ribosome scanning of 5' UTR sequences and the translation of numerous ORFs (Calviello et

332 al., 2016; Ji et al., 2015; Ruiz-Orera et al., 2014), especially in the 5' end of the RNA (Ingolia et

333 al., 2014). Population analyses on single nucleotide polymorphisms led to the conclusion that

334 many ORFs produce neutral peptides, but some of them are conserved across different species

335 and might translate functional small peptides and proteins (Bazzini et al., 2014; Ruiz-Orera et al.,

336 2018). Small peptides are usually difficult to detect as the small size may hinder the detection by

337 proteomics (Slavoff et al., 2013). As a result, some annotated IncRNAs have only recently been

338 found to translate small functional proteins. This includes cases of IncRNAs previously reported

339 to be functional at the non-coding level, as Tunar/Megamind (Lin et al., 2014) or MrIn (Anderson

340 et al., 2015; Yu et al., 2017). Here we detected an enrichment of ORFs in conserved regions,

341 which is biased towards the 5'end of the transcript, with antisense IncRNAs showing the highest

342 enrichment. We found at least 10 cases in which the encoded peptide is likely to be functional,

343 and which deserve further investigations. In many other cases the ORFs translated peptides that

344 did not showed signs of functionality, as recently observed for many species- and lineage-

345 specific transcripts (Ruiz-Orera et al., 2018). It is also possible that, in some cases, the ORF

346 may have differed extensively between mouse and human due to the rewire of non-conserved 3'

347 end exons (Almada et al., 2013). The results are also consistent with the hypothesis that some

348 some translated sequences in IncRNAs might be regulatory ORFs that influence the stability of

349 the transcript (Carlevaro-Fita et al., 2016; Johnstone et al., 2016); in some cases the putative

350 regulatory ORFs may derive from ancient protein-coding genes (Hezroni et al., 2017). In contrast

351 to IncRNAs, most small proteins translated by protein-coding genes showed evidence of

352 selection at the protein level. This included several recently discovered micropeptides, such as

353 Nbdy (D'Lima et al., 2017). As the ribosome profiling data we analyzed was from neural tissues,

354 the newly discovered micropeptides are likely to be enriched in neural functions. The analysis of

355 different tissues and conditions might reveal new functional small peptides that are not

356 expressed or translated in hippocampus. For example, we did not find expression of some

357 recently characterized small functional peptides such as Apela (Pauli et al., 2014), Spaar 
358 (Matsumoto et al., 2016), Dworf (Nelson et al., 2016), or Mymx (Zhang et al., 2017), which are

359 expressed in other tissues (Ruiz-Orera et al., 2018).

361 Although we found many translated ORFs in IncRNAs, many Ribo-Seq reads were distributed

362 along the transcript with low three-frame periodicity and uniformity, two parameters that are used

363 to predict protein translation (Ji et al., 2015). These reads are often the result of non-ribosomal

364 RNA-protein interactions and do not correspond to true 80S footprints. Two different methods

365 have been proposed for the identification of such ribonucleoprotein particles (RNP) signatures:

366 FLOSS, which is based on deviations from the expected RNA length covered by ribosomes

367 (Ingolia et al., 2014) and Rfoot, which selects regions on the basis of low uniformity of the reads

368 (Ji et al., 2016). We reasoned that protein-RNA interactions should display the two types of

369 signatures to be sufficiently reliable, and designed a specific pipeline that integrated the two

370 approaches. The method selected 21 functionally characterized IncRNAs, including intergenic

371 loci as Malat, Neat1, or TERC, and 19 loci known to host small RNA elements, such as

372 microRNAs or snoRNAs, as well as 44 new unknown candidates. These IncRNAs will be an

373 interesting resource for characterizing novel functional RNA-protein interactions, as they

374 displayed the same level of sequence constraints than functionally characterized IncRNAs. We

375 also found a significant number of RNPs within non-conserved regions; this could be due to

376 promiscuous RNA-protein interactions (Davidovich et al., 2013), the existence of young

377 functional IncRNAs (Durruthy-Durruthy et al., 2015; Heinen et al., 2009; Rigoutsos et al., 2017),

378 or IncRNAs that only contain repetitive, very small, or poorly conserved sequences, as observed

379 for the functionally characterized ncRNA Firre (Hacisuleyman et al., 2014) or for some

380 secondary structure elements detected in Neat1 (Lin et al., 2018).

382 This study has shown that mouse and human conserved IncRNA sequences show significant 383 evolutionary constraints and a more than two-fold enrichment in ribosome profiling (Ribo-Seq)

384 signatures with respect to non-conserved regions. This includes a number of putative functional 385 micropeptides as well as IncRNAs that contain protein-RNA interaction domains. When we 
consider translated open reading frames, protein-RNA interaction signatures, putative promoter regions and overlapping antisense exons, our analysis covers $77.4 \%$ of the annotated mouse IncRNA sequences with significant homology to human transcripts (Additional file 1: Figure S12). This study integrates disparate data into a common evolutionary framework and builds testable hypotheses about the functions of many IncRNAs.

\section{EXPERIMENTAL PROCEDURES}

\section{Identification of conserved and non-conserved regions in the mouse transcriptome}

We retrieved genome sequences, gene annotations, and regulatory regions (core promoters elements) from Ensembl v.89 for mouse (Flicek et al., 2013). We excluded pseudogenes and sense intronic IncRNAs, as the latter could represent unannotated regions of protein-coding genes. In order to avoid spurious conservation matches due to the presence of repeats and transposable elements, we masked repetitive sequences with RepeatMasker (Smit, AFA, Hubley, R \& Green). The masked regions comprised $11.30 \%$ of codRNA and $11.56 \%$ of IncRNA total sequence. We retained those sequences that had a minimum length of 200 nucleotides and a non-masked sequence length of at least 100 nucleotides or $25 \%$ of the total transcript length.

We run BLASTN (Altschul et al., 1997) of the mouse annotated genes against a human transcriptome sequenced at high depth, and comprising both annotated and novel transcripts, from a previous study (Ruiz-Orera et al. 2018). The human transcriptome can be downloaded from http://dx.doi.org/10.6084/m9.figshare.4702375. The BLASTN parameters employed were: -evalue $10^{-5}$, -strand plus, -max_target_seqs 15, -window_size 12. Next, we defined 'conserved regions' in mouse transcripts as the ones showing significant sequence similarity (E-value $<10^{-5}$ ) in the human transcriptome. Results were consistent when modifying e-value parameters, as the number of conserved IncRNAs only increased a $4.65 \%$ when relaxing the parameter (E-value < $10^{-4}$ ) or decreased a $3.36 \%$ when making the parameter more stringent $\left(\mathrm{E}\right.$-value $\left.<10^{-6}\right)$. 
415 Overlapping BLASTN hits from different transcripts were merged, so every gene had a unique

416 set of conserved non-redundant regions. We defined the gene as codRNA if at least one of the 417 isoforms was protein-coding, otherwise it was defined as IncRNA. We discarded 368 IncRNAs

418 that had homology to sequences annotated as coding in human, as their status was unclear and

419 they might represent unnanotated proteins or pseudogenized IncRNAs. Additionally, if two 420 conserved regions were separated by less than 100 nucleotides we merged them. This was

421 justified by the observation that less than $5 \%$ of the annotated coding sequences had internal

422 gaps longer than 100 nucleotides. Using this criterion we were able to recover $>95 \%$ of total 423 coding sequence length for the cases in which at least one conserved region was found in the 424 translated sequence. This last step had only a minor effect on the median length of the 425 conserved regions in IncRNAs (from 136 nt to 163 nt, Additional file 1: Figure S1). The method 426 identified conserved regions in 19,779 out of 21,416 protein-coding genes (codRNAs) and 1,594 427 out of 9,734 IncRNAs. Analysis of mouse-human genomic synteny alignments from UCSC 428 (Schwartz et al., 2003) indicated that about 80\% of the mouse IncRNA conserved regions could 429 be aligned to human syntenic regions, whereas this fraction decreased to about $50 \%$ for non430 conserved regions, including many tandem repeats that were masked by BLAST and that are 431 often over-represented in whole-genome alignments (Hezroni et al., 2015).

We quantified the overlap of conserved and non-conserved regions in codRNAs and IncRNAs

434 with regions annotated as promoters in Ensembl, which covered about $1.62 \%$ of the genome.

435 These regions are defined by performing peak calling from data corresponding to open 436 chromatin, histone modification, and transcripiton factor binding assays for several cell lines and 437 tissues (Zerbino et al., 2015). 
441 In order to test for selection in the aligned mouse and human sequences, we simulated the

442 evolution of sequences along the mouse and human lineages in the absence of selection with

443 Rose (Stoye et al., 1998). As starting sequences we used the annotated mouse IncRNA

444 sequences, as this allowed us to control for sequence composition and GC content. We used

445 the following parameters: HKY model with a TT ratio of 4.26; mouse branch mean substitution

4460.34 and indel rate $0.018 \times 2$; human branch mean substitution 0.17 and indel rate $0.009 \times 2$; indel

447 function: $[.50, .18, .10, .08, .06, .04, .04]$. Mean substitutions and rate of insertions and deletions

448 values were based on previous estimates (Consortium, 2002; Lunter, 2007; Ogurtsov et al.,

449 2004), using a twofold higher mutation rate in mouse than in human.

After the simulations we run BLASTN, using the same conditions as for real sequences, and

452 recovered the alignments. Up to $59.6 \%$ of the mouse simulated sequences had at least one 453 match in the set of human simulated sequences. This corresponded to the $20.8 \%$ of the total 454 sequence length.

\section{Calculation of substitution rates}

We estimated the number of substitutions per site ( $k$ or $k_{0}$ ) in BLAST alignments using the 459 maximum likelihood method 'baseml' from the PAML package (Yang, 2007) with model 7 (REV).

460 If a position was covered by several BLAST hits we chose the one with the lowest E-value. We 461 discarded $k$ values higher than 5 , as they might represent computational artifacts. As we 462 observed that $k$ values deviate from neutrality in simulations of neutrally evolved sequences with 463 short length, we also computed a normalized substitution rate $k_{d} / k_{e}$, being $k_{e}$ the expected 464 neutral rate according to the length of the region after modeling a log-linear regression model for 465 short (<300 nt) and long (>= $300 \mathrm{nt})$ neutrally evolved sequences separately:

$466 \log \left(k_{p} ; L>=300\right)=-0.468900-L \times 7.865 \times 10^{-5}$

$467 \quad \log \left(k_{p} ; L<300\right)=-1.562833+L \times 0.003879$

468 This model was statistically significant for short and long sequences (T-test, $p$-value $<0.05)$. 
470 Classifications of genes based on genomic location or small RNA content

471 Up to $20 \%$ of total sequence length in IncRNAs had exonic overlaps with other genes in the

472 antisense strand. Therefore, we divided conserved and non-conserved regions into 'overlapping'

473 and 'non-overlapping', depending on whether the region was overlapping with a conserved

474 feature in the other strand (detected by BLAST or annotated as conserved in human in Ensembl

475 Compara). After classifying regions in these 4 different categories, we finally discarded regions

476 shorter than $30 \mathrm{nt}$ and were not considered either as part of the gene, as they might be artifact

477 gaps from homology searches.

479 Finally, we classified genes in three different categories: ncRNA host, in the case of genes with

480 annotated small RNAs in the exonic structure and/or being annotated microRNA or small RNA

481 host; antisense, in the case of genes having at least one overlapping region, being expressed

482 from bidirectional promoters (closer than $2 \mathrm{~kb}$ to an annotated TSS from a antisense protein-

483 coding gene) and/or being annotated as antisense in Ensembl; or intergenic otherwise.

\section{Analysis of RNA-seq and Ribo-Seq coverage}

486 We used RNA-seq and ribosome profiling data (Ribo-Seq) from mouse hippocampus obtained

487 from Gene Expression Omnibus under accession number GSE72064 (Cho et al., 2015). We

488 merged sequencing replicates to increase the power to detect translated ORFs. We removed

489 reads mapping to annotated rRNAs and tRNAs. Next, we mapped Ribo-Seq (361 million

490 mapped reads) and RNA-seq reads (435 million mapped reads) to the mouse genome (mm10)

491 using Bowtie (v. 0.12.7, parameters -k 1 -m 20 -n 1 --best -strata) (Langmead et al., 2009) and

492 we extracted P-sites corresponding to Ribo-Seq reads as done in a previous study (Ruiz-Orera

493 et al., 2018). For comparison, we analyzed Ribo-Seq data from rat brain (rn6, 373 million

494 mapped reads) and human brain (hg19, 50 million mapped reads) obtained from Gene

495 Expression Omnibus under accession numbers GSE66715 (Cho et al., 2015) and GSE51424

496 (Gonzalez et al., 2014). 
498 Next, we assigned strand-specific mouse reads to the different transcript regions if at least $1 \mathrm{bp}$

499 (RNA-seq) or the computed P-site (Ribo-Seq) spanned the corresponding region. We defined

500 two metrics: a per-base coverage metric based on the number of reads spanning the region per

501 kilobase, and a total coverage based on the percentage of sequence covered by reads.

503 For genes expressed at very low levels the Ribo-Seq signal may become undetectable. In order

504 to account for this we selected a RNA-seq coverage threshold in which the number of false

505 negatives (annotated coding sequences not covered by RiboSeq reads) was lower than $5 \%$

506 (Additional file 1: Figure S13, RNA-Seq coverage in region $\geq 56.38$ reads/kb). In conserved

507 genes, at least one of the conserved regions had to show a coverage above the threshold, or the

508 whole gene was considered as not expressed. Finally, we eliminated 192 IncRNAs located within

$5094 \mathrm{~kb}$ from a sense protein-coding gene and/or with evidence of being part of the same gene using

510 RNA-Seq data, these IncRNAs may have been unannotated UTRs.

\section{ORF translation in conserved and non-conserved regions}

513 We predicted all translated ORFs (ATG to STOP) with a minimum length of 9 amino acids in the

514 transcripts with RibORF (v.0.1) (Ji et al., 2015). Only ORFs with a minimum of 10 mapped Ribo-

515 Seq reads were considered. We used the same score cut-off as in our previosus study ( $\geq 0.7)$,

516 which had a reported false positive rate of $3.30-4.16 \%$ and a false negative rate of $2.54 \%$ (Ruiz-

517 Orera et al., 2018).

519 Next, we assigned translated ORFs to the different defined regions if at least $10 \%$ of the

520 translated sequence spanned a single region. When multiple ORFs spanned one region, we

521 selected the longest one as representative of the region. Consequently, a single ORF could span

522 multiple gene regions, including also discarded ones because of the short length or low

523 expression. 


\section{dN/dS analysis in translated ORFs}

We used the UCSC tool liftOver (-minMatch=0.75) (Tyner et al., 2017) to extract the corresponding ORF genomic coordinates in human. For the cases in which we found a matching region, we aligned the ORFs with PRANK (Loytynoja and Goldman, 2005) and we checked how many of these sequences were complete and had the same start-stop codon structure in human, with a resulting coding sequence having at least $50 \%$ of the length of the ORF in mouse. Besides, alignment should not contain more than $33 \%$ of gaps and aligned length should be longer than 10 amino acids. The remaining ORFs were considered to be truncated.

Next, we used CODEML of the PAML package (Yang, 2007) to compute a dN/dS ratio per complete ORF and we tested whether this ratio was significantly different from 1 by running a fixed omega model. We found 10 mouse and human conserved ORFs in IncRNAs with dN/dS significantly lower than 1 , with an adjusted $p$-value $<0.05$.

\section{PhyloP codon analysis in translated ORFs}

We used the GenomicScores package (v. 1.2.2) available at Bioconductor (Puigdevall and Castelo, 2018) to compute the average PhyloP score per codon position $(+1,+2,+3)$ in different sets of translated ORFs. PhyloP is a set of phylogenetic $p$-values for multiple alignments of 59 vertebrate genomes to the mouse genome. GenomicScores round PhyloP scores using a lossy compresion algorithm. We checked if there was a lower conservation in the third position, as it has been observed in functional proteins (Pollard et al., 2010) due to the degeneracy of the third nucleotide in many codons.

\section{Analysis of RNA-protein complexes}

We used Rfoot (v.0.1) and FLOSS to analyze how many regions in IncRNAs might be involved in RNA-protein complexes (RNPs). Rfoot is a tool that analyzes Ribo-Seq data to identify regions that lack read periodicity and have low read uniformity, and which may correspond to nonribosomal ribonucleoprotein associates ( $\mathrm{Ji}$ et al., 2016). FLOSS is a tool that analyzes the 
553 distribution of Ribo-Seq read lengths and measures the magnitude of disagreement between

554 distributions to separate ribosome and non-ribosome signals (Ingolia et al., 2014).

556 We identified putative RNA-protein interaction regions by selecting 60nt windows showing

557 uniformity $<0.6$ with a minimum of 10 reads, as done in the original study (Ji et al., 2015).

558 Moreover, we subtracted predicted ORF sequences with a RibORF score $\geq 0.5$ and/or read

559 periodicity $\geq 0.66$. As ribosome-protected UTR regions could be present in the selected regions,

560 we computed a FLOSS score per region and we defined as RNPs the ones in which the

561 divergence score from ribosome-protected regions was $\geq 0.35$. This threshold was selected

562 because only $5 \%$ of CDS regions showed a score above 0.35 . Subsequently, we merged

563 overlapping regions into a single RNP. This combined approach found RNP associations in 95\%

564 of a control set of snRNAs and snoRNAs with 10 or more Ribo-Seq reads, and in only $20 \%$ of

565 the 5' UTRs with the same number of Ribo-Seq reads.

566

567 Evidence of functionality in IncRNAs

568 We obtained a list of 30 functional mouse IncRNAs expressed in hippocampus by selecting all

569 cases present in IncRNAdb (Quek et al., 2014) and adding four additional known IncRNAs:

570 Pantr1 (Goff et al., 2015), Firre (Hacisuleyman et al., 2014), TERC (Feng et al., 1995), and

571 Norad (Lee et al., 2016).

572

573 Statistical tests and plots

574 Plots and statistics was performed with the R package (Team, 2013).

\section{SUPPLEMENTAL INFORMATION}

578 Additional file 1: File with supplementary information (tables and figures). 
579 Additional file 2: Excel file with properties of the defined IncRNA regions and genes, a list of

580 functionally characterized IncRNAs, and peptide sequences in mouse and human for the 10

581 functional micropeptides.

582 Additional file 3: BED file with the coordinates of the IncRNA regions ('exon' field), the 492

583 translated sequences ('ORF' field) and the defined RNA-protein interactions ('RNP' field).

585 ACKNOWLEDGMENTS AND FUNDING

586 The work was funded by grant BFU2015-65235-P from Ministerio de Economía e Innovación

587 (Spanish Government) co-funded by FEDER (EU), and by grant 2014SGR1121 from Agència de

588 Gestió d'Ajuts Universitaris i de Recerca (AGAUR, Generalitat de Catalunya).

\section{AUTHOR CONTRIBUTIONS}

591 J.R-O. and M.M.A. conceived the study, interpreted the data and wrote the paper. J.R-O.

592 performed the analyses. M.M.A. coordinated the study. All authors read and approved the final

593 manuscript.

\section{DECLARATION OF INTERESTS}

596 The authors declare that they have no competing interests.

\section{REFERENCES}

599 Almada, A.E., Wu, X., Kriz, A.J., Burge, C.B., and Sharp, P.A. (2013). Promoter directionality is 600 controlled by U1 snRNP and polyadenylation signals. Nature 499, 360-363.

601 Altschul, S.F., Madden, T.L., Schäffer, A.A., Zhang, J., Zhang, Z., Miller, W., and Lipman, D.J.

602 (1997). Gapped BLAST and PSI-BLAST: a new generation of protein database search

603 programs. Nucleic Acids Res. 25, 3389-3402.

604 Anderson, D.M., Anderson, K.M., Chang, C.-L., Makarewich, C.A., Nelson, B.R., McAnally, J.R., 605 Kasaragod, P., Shelton, J.M., Liou, J., Bassel-Duby, R., et al. (2015). A micropeptide encoded by 606 a putative long noncoding RNA regulates muscle performance. Cell 160, 595-606. 
Aspden, J.L., Eyre-Walker, Y.C., Philips, R.J., Amin, U., Mumtaz, M.A.S., Brocard, M., and Couso, J.-P. (2014). Extensive translation of small ORFs revealed by Poly-Ribo-Seq. Elife e03528.

Bazzini, A.A., Johnstone, T.G., Christiano, R., Mackowiak, S.D., Obermayer, B., Fleming, E.S., Vejnar, C.E., Lee, M.T., Rajewsky, N., Walther, T.C., et al. (2014). Identification of small ORFs in vertebrates using ribosome footprinting and evolutionary conservation. EMBO J.

Brosius, J. (2005). Waste not, want not--transcript excess in multicellular eukaryotes. Trends Genet. 21, 287-288.

Buck-Koehntop, B.A., Mascioni, A., Buffy, J.J., and Veglia, G. (2005). Structure, dynamics, and membrane topology of stannin: a mediator of neuronal cell apoptosis induced by trimethyltin chloride. J. Mol. Biol. 354, 652-665.

Cabili, M.N., Trapnell, C., Goff, L., Koziol, M., Tazon-Vega, B., Regev, A., and Rinn, J.L. (2011). Integrative annotation of human large intergenic noncoding RNAs reveals global properties and specific subclasses. Genes Dev. 25, 1915-1927.

Calviello, L., Mukherjee, N., Wyler, E., Zauber, H., Hirsekorn, A., Selbach, M., Landthaler, M., Obermayer, B., and Ohler, U. (2016). Detecting actively translated open reading frames in ribosome profiling data. Nat Meth 13, 165-170.

Carlevaro-Fita, J., Rahim, A., Guigo, R., Vardy, L.A., and Johnson, R. (2016). Cytoplasmic long noncoding RNAs are frequently bound to and degraded at ribosomes in human cells. RNA 22, 867-882.

Carninci, P., Kasukawa, T., Katayama, S., Gough, J., Frith, M.C., Maeda, N., Oyama, R., Ravasi, T., Lenhard, B., Wells, C., et al. (2005). The transcriptional landscape of the mammalian genome. Science 309, 1559-1563.

Castaneda, J., Genzor, P., van der Heijden, G.W., Sarkeshik, A., Yates, J.R. 3rd, Ingolia, N.T., and Bortvin, A. (2014). Reduced pachytene piRNAs and translation underlie spermiogenic arrest in Maelstrom mutant mice. EMBO J. 33, 1999-2019.

Chew, G.-L., Pauli, A., Rinn, J.L., Regev, A., Schier, A.F., and Valen, E. (2013). Ribosome profiling reveals resemblance between long non-coding RNAs and 5' leaders of coding RNAs. Development 140, 2828-2834.

Cho, J., Yu, N.-K., Choi, J.-H., Sim, S.-E., Kang, S.J., Kwak, C., Lee, S.-W., Kim, J., Choi, D. II, Kim, V.N., et al. (2015). Multiple repressive mechanisms in the hippocampus during memory formation. Science 350, 82-87.

Consortium, M.G.S. (2002). Initial sequencing and comparative analysis of the mouse genome. Nature 420, 520.

Consortium, T.E.P., Birney, E., Stamatoyannopoulos, J.A., Dutta, A., Guigo, R., and Gingeras, T.R. (2007). Identification and analysis of functional elements in $1\{\%\}$ of the human genome by the ENCODE pilot project. Nature 447, 799-816.

D'Lima, N.G., Ma, J., Winkler, L., Chu, Q., Loh, K.H., Corpuz, E.O., Budnik, B.A., LykkeAndersen, J., Saghatelian, A., and Slavoff, S.A. (2017). A human microprotein that interacts with the mRNA decapping complex. Nat Chem Biol 13, 174-180. 
647 Davidovich, C., Zheng, L., Goodrich, K.J., and Cech, T.R. (2013). Promiscuous RNA binding by

648 Polycomb Repressive Complex 2. Nat. Struct. Mol. Biol. 20, 1250-1257.

649 Derrien, T., Johnson, R., Bussotti, G., Tanzer, A., Djebali, S., Tilgner, H., Guernec, G., Martin, D., 650 Merkel, A., Knowles, D.G., et al. (2012). The GENCODE v7 catalog of human long noncoding 651 RNAs: analysis of their gene structure, evolution, and expression. Genome Res. 22, 1775-1789.

652 Durruthy-Durruthy, J., Sebastiano, V., Wossidlo, M., Cepeda, D., Cui, J., Grow, E.J., Davila, J., 653 Mall, M., Wong, W.H., Wysocka, J., et al. (2015). The primate-specific noncoding RNA HPAT5

654 regulates pluripotency during human preimplantation development and nuclear reprogramming.

655 Nat. Genet. 48, 44.

656 Feng, J., Funk, W.D., Wang, S.S., Weinrich, S.L., Avilion, A.A., Chiu, C.P., Adams, R.R., Chang, 657 E., Allsopp, R.C., and Yu, J. (1995). The RNA component of human telomerase. Science 269, 658 1236-1241.

659 Flicek, P., Ahmed, I., Amode, M.R., Barrell, D., Beal, K., Brent, S., Carvalho-Silva, D., Clapham, 660 P., Coates, G., Fairley, S., et al. (2013). Ensembl 2013. Nucleic Acids Res. 41, D48-55.

661 Goff, L.A., Groff, A.F., Sauvageau, M., Trayes-Gibson, Z., Sanchez-Gomez, D.B., Morse, M., 662 Martin, R.D., Elcavage, L.E., Liapis, S.C., Gonzalez-Celeiro, M., et al. (2015). Spatiotemporal 663 expression and transcriptional perturbations by long noncoding RNAs in the mouse brain. Proc. 664 Natl. Acad. Sci. U. S. A. 112, 6855-6862.

665 Gong, C., Popp, M.W.-L., and Maquat, L.E. (2012). Biochemical analysis of long non-coding 666 RNA-containing ribonucleoprotein complexes. Methods 58, 88-93. Gonzalez, C., Sims, J.S., Hornstein, N., Mela, A., Garcia, F., Lei, L., Gass, D. a, Amendolara, B., 668 Bruce, J.N., Canoll, P., et al. (2014). Ribosome profiling reveals a cell-type-specific translational 669 landscape in brain tumors. J. Neurosci. 34, 10924-10936.

670 Guttman, M., Amit, I., Garber, M., French, C., Lin, M.F., Feldser, D., Huarte, M., Zuk, O., Carey, 671 B.W., Cassady, J.P., et al. (2009). Chromatin signature reveals over a thousand highly

672 conserved large non-coding RNAs in mammals. Nature 458, 223-227.

673 Guttman, M., Russell, P., Ingolia, N.T., Weissman, J.S., and Lander, E.S. (2013). Ribosome 674 profiling provides evidence that large noncoding RNAs do not encode proteins. Cell 154, 240 675251.

676 Hacisuleyman, E., Goff, L.A., Trapnell, C., Williams, A., Henao-Mejia, J., Sun, L., McClanahan, 677 P., Hendrickson, D.G., Sauvageau, M., Kelley, D.R., et al. (2014). Topological organization of 678 multichromosomal regions by the long intergenic noncoding RNA Firre. Nat. Struct. Mol. Biol. 21, 679 198-206.

680 Han, S.P., Tang, Y.H., and Smith, R. (2010). Functional diversity of the hnRNPs: past, present 681 and perspectives. Biochem. J. 430, 379-392.

682 He, Y., Ding, Y., Zhan, F., Zhang, H., Han, B., Hu, G., Zhao, K., Yang, N., Yu, Y., Mao, L., et al. 683 (2015). The conservation and signatures of lincRNAs in Marek's disease of chicken. Sci. Rep. 5, 68415184.

685 Hedges, S.B., Marin, J., Suleski, M., Paymer, M., and Kumar, S. (2015). Tree of life reveals 686 clock-like speciation and diversification. Mol. Biol. Evol. 32, 835-845. 
van Heesch, S., van Iterson, M., Jacobi, J., Boymans, S., Essers, P.B., de Bruijn, E., Hao, W., Macinnes, A.W., Cuppen, E., and Simonis, M. (2014). Extensive localization of long noncoding RNAs to the cytosol and mono- and polyribosomal complexes. Genome Biol. 15, R6.

Heinen, T.J. a J., Staubach, F., Häming, D., and Tautz, D. (2009). Emergence of a new gene from an intergenic region. Curr. Biol. 19, 1527-1531.

Hezroni, H., Koppstein, D., Schwartz, M.G., Avrutin, A., Bartel, D.P., and Ulitsky, I. (2015). Principles of Long Noncoding RNA Evolution Derived from Direct Comparison of Transcriptomes in 17 Species. Cell Rep. 1-13.

Hezroni, H., Ben-Tov Perry, R., Meir, Z., Housman, G., Lubelsky, Y., and Ulitsky, I. (2017). A subset of conserved mammalian long non-coding RNAs are fossils of ancestral protein-coding genes. Genome Biol. 18, 162.

Hon, C.-C., Ramilowski, J.A., Harshbarger, J., Bertin, N., Rackham, O.J.L., Gough, J., Denisenko, E., Schmeier, S., Poulsen, T.M., Severin, J., et al. (2017). An atlas of human long non-coding RNAs with accurate 5' ends. Nature 543, 199-204.

Ingolia, N.T., Ghaemmaghami, S., Newman, J.R.S., and Weissman, J.S. (2009). Genome-wide analysis in vivo of translation with nucleotide resolution using ribosome profiling. Science 324 , 218-223.

Ingolia, N.T., Lareau, L.F., and Weissman, J.S. (2011). Ribosome profiling of mouse embryonic stem cells reveals the complexity and dynamics of mammalian proteomes. Cell 147, 789-802.

Ingolia, N.T., Brar, G.A., Stern-Ginossar, N., Harris, M.S., Talhouarne, G.J.S., Jackson, S.E., Wills, M.R., and Weissman, J.S. (2014). Ribosome Profiling Reveals Pervasive Translation Outside of Annotated Protein-Coding Genes. Cell Rep. 8, 1365-1379.

Ji, Z., Song, R., Regev, A., and Struhl, K. (2015). Many IncRNAs, 5'UTRs, and pseudogenes are translated and some are likely to express functional proteins. Elife 4, e08890.

Ji, Z., Song, R., Huang, H., Regev, A., and Struhl, K. (2016). Transcriptome-scale RNasefootprinting of RNA-protein complexes. Nat. Biotechnol. 34, 410-413.

Johnstone, T.G., Bazzini, A.A., and Giraldez, A.J. (2016). Upstream ORFs are prevalent translational repressors in vertebrates. EMBO J.

Juntawong, P., Girke, T., Bazin, J., and Bailey-Serres, J. (2014). Translational dynamics revealed by genome-wide profiling of ribosome footprints in Arabidopsis. Proc. Natl. Acad. Sci. U. S. A. 111, E203-12.

Kapranov, P., Cheng, J., Dike, S., Nix, D.A., Duttagupta, R., Willingham, A.T., Stadler, P.F., Hertel, J., Hackermüller, J., Hofacker, I.L., et al. (2007). RNA maps reveal new RNA classes and a possible function for pervasive transcription. Science 316, 1484-1488.

Kapusta, A., and Feschotte, C. (2014). Volatile evolution of long noncoding RNA repertoires: mechanisms and biological implications. Trends Genet. 30, 439-452.

Kutter, C., Watt, S., Stefflova, K., Wilson, M.D., Goncalves, A., Ponting, C.P., Odom, D.T., and Marques, A.C. (2012). Rapid turnover of long noncoding RNAs and the evolution of gene expression. PLoS Genet. 8, e1002841. 
Langmead, B., Trapnell, C., Pop, M., and Salzberg, S.L. (2009). Ultrafast and memory-efficient alignment of short DNA sequences to the human genome. Genome Biol. 10, R25.

Lee, S., Kopp, F., Chang, T.-C., Sataluri, A., Chen, B., Sivakumar, S., Yu, H., Xie, Y., and Mendell, J.T. (2016). Noncoding RNA NORAD Regulates Genomic Stability by Sequestering PUMILIO Proteins. Cell 164, 69-80.

Lepoivre, C., Belhocine, M., Bergon, A., Griffon, A., Yammine, M., Vanhille, L., Zacarias-Cabeza, J., Garibal, M.-A., Koch, F., Maqbool, M.A., et al. (2013). Divergent transcription is associated with promoters of transcriptional regulators. BMC Genomics 14, 914.

Li, D., and Yang, M.Q. (2017). Identification and characterization of conserved IncRNAs in human and rat brain. BMC Bioinformatics 18, 489.

Li, W., Notani, D., and Rosenfeld, M.G. (2016). Enhancers as non-coding RNA transcription units: recent insights and future perspectives. Nat. Rev. Genet. 17, 207.

Lin, N., Chang, K.-Y., Li, Z., Gates, K., Rana, Z.A., Dang, J., Zhang, D., Han, T., Yang, C.-S., Cunningham, T.J., et al. (2014). An Evolutionarily Conserved Long Noncoding RNA TUNA Controls Pluripotency and Neural Lineage Commitment. Mol. Cell 53, 1005-1019.

Lin, Y., Schmidt, B.F., Bruchez, M.P., and McManus, C.J. (2018). Structural analyses of NEAT1 IncRNAs suggest long-range RNA interactions that may contribute to paraspeckle architecture. Nucleic Acids Res. 46, 3742-3752.

Liu, J., Jung, C., Xu, J., Wang, H., Deng, S., Bernad, L., Arenas-Huertero, C., and Chua, N.-H. (2012). Genome-wide analysis uncovers regulation of long intergenic noncoding RNAs in Arabidopsis. Plant Cell 24, 4333-4345.

Loytynoja, A., and Goldman, N. (2005). An algorithm for progressive multiple alignment of sequences with insertions. Proc. Natl. Acad. Sci. U. S. A. 102, 10557-10562.

Lunter, G. (2007). Probabilistic whole-genome alignments reveal high indel rates in the human and mouse genomes. Bioinformatics 23, i289-i296.

Magny, E.G., Pueyo, J.I., Pearl, F.M.G., Cespedes, M.A., Niven, J.E., Bishop, S. a, and Couso, J.P. (2013). Conserved regulation of cardiac calcium uptake by peptides encoded in small open reading frames. Science 341, 1116-1120.

Marques, A.C., and Ponting, C.P. (2009). Catalogues of mammalian long noncoding RNAs: modest conservation and incompleteness. Genome Biol. 10, R124-R124.

Matsumoto, A., Pasut, A., Matsumoto, M., Yamashita, R., Fung, J., Monteleone, E., Saghatelian, A., Nakayama, K.I., Clohessy, J.G., and Pandolfi, P.P. (2016). mTORC1 and muscle regeneration are regulated by the LINC00961-encoded SPAR polypeptide. Nature 541, 228.

Mohammadin, S., Edger, P.P., Pires, J.C., and Schranz, M.E. (2015). Positionally-conserved but sequence-diverged: identification of long non-coding RNAs in the Brassicaceae and Cleomaceae. BMC Plant Biol. 15, 217.

Necsulea, A., Soumillon, M., Warnefors, M., Liechti, A., Daish, T., Zeller, U., Baker, J.C., Grützner, F., and Kaessmann, H. (2014). The evolution of IncRNA repertoires and expression patterns in tetrapods. Nature. 
Nelson, B.R., Makarewich, C.A., Anderson, D.M., Winders, B.R., Troupes, C.D., Wu, F., Reese, A.L., McAnally, J.R., Chen, X., Kavalali, E.T., et al. (2016). A peptide encoded by a transcript annotated as long noncoding RNA enhances SERCA activity in muscle. Science 351, 271-275.

Neme, R., and Tautz, D. (2016). Fast turnover of genome transcription across evolutionary time exposes entire non-coding DNA to de novo gene emergence. Elife 5, e09977.

Ogurtsov, A.Y., Sunyaev, S., and Kondrashov, A.S. (2004). Indel-Based Evolutionary Distance and Mouse-Human Divergence. Genome Res. 14, 1610-1616.

Okazaki, Y., Furuno, M., Kasukawa, T., Adachi, J., Bono, H., Kondo, S., Nikaido, I., Osato, N., Saito, R., Suzuki, H., et al. (2002). Analysis of the mouse transcriptome based on functional annotation of 60,770 full-length cDNAs. Nature $420,563-573$.

Pauli, A., Valen, E., Lin, M.F., Garber, M., Vastenhouw, N.L., Levin, J.Z., Fan, L., Sandelin, A., Rinn, J.L., Regev, A., et al. (2012). Systematic identification of long noncoding RNAs expressed during zebrafish embryogenesis. 577-591.

Pauli, A., Norris, M.L., Valen, E., Chew, G.-L., Gagnon, J.A., Zimmerman, S., Mitchell, A., Ma, J., Dubrulle, J., Reyon, D., et al. (2014). Toddler: an embryonic signal that promotes cell movement via Apelin receptors. Science 343, 1248636.

Pegueroles, C., and Gabaldón, T. (2016). Secondary structure impacts patterns of selection in human IncRNAs. BMC Biol. 14, 1-13.

Pollard, K.S., Hubisz, M.J., Rosenbloom, K.R., and Siepel, A. (2010). Detection of nonneutral substitution rates on mammalian phylogenies. Genome Res. 20.

Polycarpou-Schwarz, M., Groß, M., Mestdagh, P., Schott, J., Grund, S.E., Hildenbrand, C., Rom, J., Aulmann, S., Sinn, H.-P., Vandesompele, J., et al. (2018). The cancer-associated microprotein CASIMO1 controls cell proliferation and interacts with squalene epoxidase modulating lipid droplet formation. Oncogene.

Ponjavic, J., Ponting, C.P., and Lunter, G. (2007). Functionality or transcriptional noise? Evidence for selection within long noncoding RNAs. Genome Res. 17, 556-565.

Ponting, C.P., Oliver, P.L., and Reik, W. (2009). Evolution and functions of long noncoding RNAs. Cell 136, 629-641.

Pueyo, J.I., Magny, E.G., Sampson, C.J., Amin, U., Evans, I.R., Bishop, S.A., and Couso, J.P. (2016). Hemotin, a Regulator of Phagocytosis Encoded by a Small ORF and Conserved across Metazoans. PLoS Biol. 14, e1002395.

Puigdevall, P., and Castelo, R. (2018). GenomicScores: seamless access to genomewide position-specific scores from R and Bioconductor. Bioinformatics bty311-bty311.

Quek, X.C., Thomson, D.W., Maag, J.L. V, Bartonicek, N., Signal, B., and Clark, M.B. (2014). IncRNAdb v2.0: expanding the reference database for functional long noncoding RNAs. Nucleic Acids Res 43.

Quinn, J.J., llik, I.A., Qu, K., Georgiev, P., Chu, C., Akhtar, A., and Chang, H.Y. (2014). Revealing long noncoding RNA architecture and functions using domain-specific chromatin isolation by RNA purification. Nat. Biotechnol. 32, 933-940. 
Ribeiro, D.M., Zanzoni, A., Cipriano, A., Delli Ponti, R., Spinelli, L., Ballarino, M., Bozzoni, I., Tartaglia, G.G., and Brun, C. (2017). Protein complex scaffolding predicted as a prevalent function of long non-coding RNAs. Nucleic Acids Res. gkx1169-gkx1169.

Rigoutsos, I., Lee, S.K., Nam, S.Y., Anfossi, S., Pasculli, B., Pichler, M., Jing, Y., RodriguezAguayo, C., Telonis, A.G., Rossi, S., et al. (2017). N-BLR, a primate-specific non-coding transcript leads to colorectal cancer invasion and migration. Genome Biol. 18, 98.

Ruiz-Orera, J., Messeguer, X., Subirana, J.A., and Alba, M.M. (2014). Long non-coding RNAs as a source of new peptides. Elife 3, 1-24.

Ruiz-Orera, J., Hernandez-Rodriguez, J., Chiva, C., Sabidó, E., Kondova, I., Bontrop, R., Marqués-Bonet, T., and Albà, M.M. (2015). Origins of De Novo Genes in Human and Chimpanzee. PLOS Genet. 11, e1005721.

Ruiz-Orera, J., Verdaguer-Grau, P., Villanueva-Cañas, J.L., Messeguer, X., and Albà, M.M. (2018). Translation of neutrally evolving peptides provides a basis for de novo gene evolution. Nat. Ecol. Evol.

Schwartz, S., Kent, W.J., Smit, A., Zhang, Z., Baertsch, R., Hardison, R.C., Haussler, D., and Miller, W. (2003). Human-mouse alignments with BLASTZ. Genome Res. 13, 103-107.

Seiler, J., Breinig, M., Caudron-Herger, M., Polycarpou-Schwarz, M., Boutros, M., and Diederichs, S. (2017). The IncRNA VELUCT strongly regulates viability of lung cancer cells despite its extremely low abundance. Nucleic Acids Res. 45, 5458-5469.

Slavoff, S.A., Mitchell, A.J., Schwaid, A.G., Cabili, M.N., Ma, J., Levin, J.Z., Karger, A.D., Budnik, B.A., Rinn, J.L., and Saghatelian, A. (2013). Peptidomic discovery of short open reading frameencoded peptides in human cells. Nat. Chem. Biol. 9, 59-64.

Smit, AFA, Hubley, R \& Green, P. RepeatMasker Open-4.0.

Stoye, J., Evers, D., and Meyer, F. (1998). Rose: generating sequence families. Bioinforma. (Formerly CABIOS).

Struhl, K. (2007). Transcriptional noise and the fidelity of initiation by RNA polymerase II. Nat. Struct. Mol. Biol. 14, 103-105.

Team, R. (2013). R Development Core Team. R A Lang. Environ. Stat. Comput.

Tyner, C., Barber, G.P., Casper, J., Clawson, H., Diekhans, M., Eisenhart, C., Fischer, C.M., Gibson, D., Gonzalez, J.N., Guruvadoo, L., et al. (2017). The UCSC Genome Browser database: 2017 update. Nucleic Acids Res. 45, D626-D634.

Ulitsky, I. (2016). Evolution to the rescue: using comparative genomics to understand long noncoding RNAs. Nat. Rev. Genet. 17, 601-614.

Ulitsky, I., and Bartel, D.P. (2013). lincRNAs: genomics, evolution, and mechanisms. Cell 154, 26-46.

Ulitsky, I., Shkumatava, A., Jan, C.H., Sive, H., and Bartel, D.P. (2011). Conserved function of lincRNAs in vertebrate embryonic development despite rapid sequence evolution. Cell 147, 1537-1550. 
842 Vakirlis, N., Hebert, A.S., Opulente, D.A., Achaz, G., Hittinger, C.T., Fischer, G., Coon, J.J., and

843 Lafontaine, I. (2018). A Molecular Portrait of De Novo Genes in Yeasts. Mol. Biol. Evol. 35, 631-

844645.

845 Wang, J., Zhang, J., Zheng, H., Li, J., Liu, D., Li, H., Samudrala, R., Yu, J., and Wong, G.K.-S.

846 (2004). Mouse transcriptome: Neutral evolution of /'non-coding/' complementary DNAs. Nature

847431.

848 Wawrzynow, A., Theibert, J.L., Murphy, C., Jona, I., Martonosi, A., and Collins, J.H. (1992).

849 Sarcolipin, the "proteolipid" of skeletal muscle sarcoplasmic reticulum, is a unique, amphipathic,

850 31-residue peptide. Arch. Biochem. Biophys. 298, 620-623.

Wiberg, R.A.W., Halligan, D.L., Ness, R.W., Necsulea, A., Kaessmann, H., and Keightley, P.D.

852 (2015). Assessing Recent Selection and Functionality at Long Noncoding RNA Loci in the Mouse

853 Genome. Genome Biol. Evol. 7, 2432-2444.

854 Wilusz, J.E., Freier, S.M., and Spector, D.L. (2008). 3' end processing of a long nuclear-retained

855 noncoding RNA yields a tRNA-like cytoplasmic RNA. Cell 135, 919-932.

856 Xing, Y.-H., Yao, R.-W., Zhang, Y., Guo, C.-J., Jiang, S., Xu, G., Dong, R., Yang, L., and Chen, 857 L.-L. (2017). SLERT Regulates DDX21 Rings Associated with Pol I Transcription. Cell 169, 664858 678.e16.

859 Yang, Z. (2007). PAML 4: phylogenetic analysis by maximum likelihood. Mol. Biol. Evol. 24, $860 \quad 1586-1591$.

861 Yu, X., Zhang, Y., Li, T., Ma, Z., Jia, H., Chen, Q., Zhao, Y., Zhai, L., Zhong, R., Li, C., et al.

862 (2017). Long non-coding RNA Linc-RAM enhances myogenic differentiation by interacting with

863 MyoD. Nat. Commun. 8, 14016.

864 Zerbino, D.R., Wilder, S.P., Johnson, N., Juettemann, T., and Flicek, P.R. (2015). The ensembl 865 regulatory build. Genome Biol. 16, 56.

866 Zhang, Q., Vashisht, A.A., O'Rourke, J., Corbel, S.Y., Moran, R., Romero, A., Miraglia, L., Zhang, 867 J., Durrant, E., Schmedt, C., et al. (2017). The microprotein Minion controls cell fusion and 868 muscle formation. Nat. Commun. 8, 15664. 


\section{FIGURE LEGENDS}

A
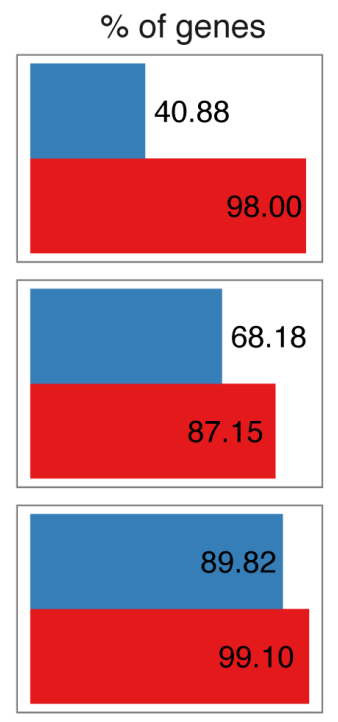

$\%$ of total sequence
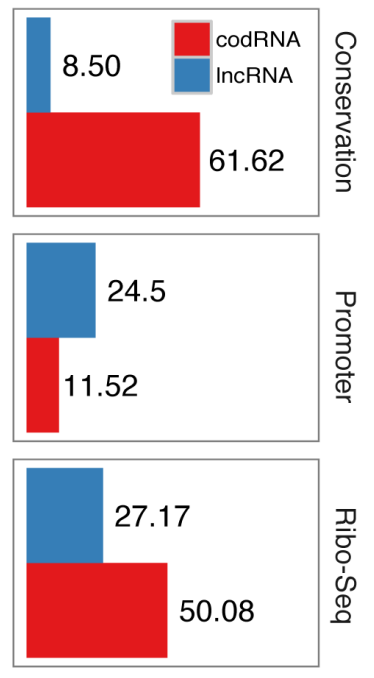

B

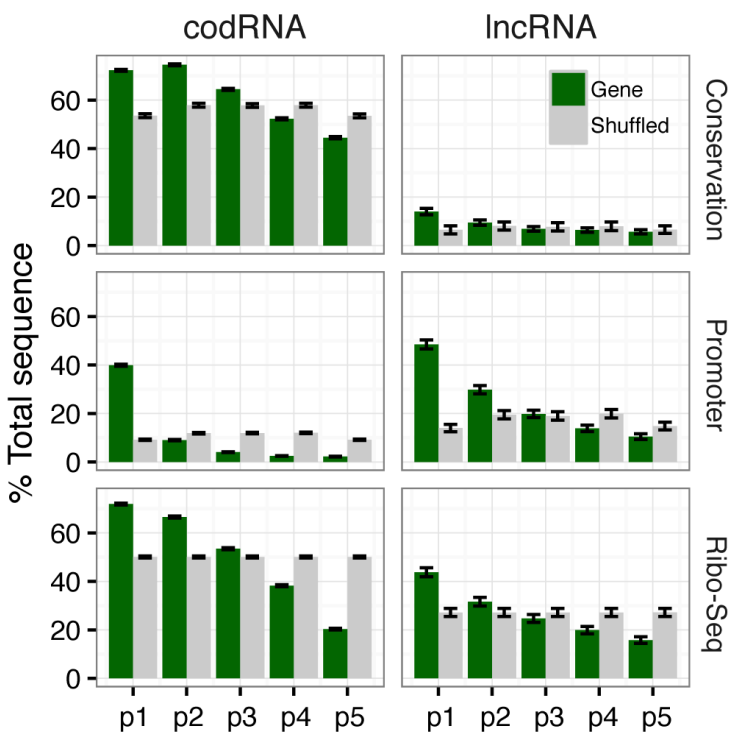

882 Figure 1. Transcriptome-wide identification of conserved sequences, promoters, and

\section{Ribo-Seq associations.}

884 A. Fraction of mouse genes that showed conservation in human using BLASTN (Conservation),

885 that overlapped with annotated promoter regions (Promoter), or that were covered by Ribo-Seq

886 reads (Ribo-Seq). The percentage of genes with at least one feature, and the total sequence

887 covered, are indicated. Data is for expressed codRNAs and IncRNAs in hippocampus

888 (sequences with a minimum RNA-Seq coverage of 56.38 reads $/ \mathrm{kb}$ ). B. Analysis of feature

889 coverage in equally-sized fractions of the genes, from $5^{\prime}(p 1)$ to $3^{\prime}(p 5)$. Grey bars represent the

890 mean proportion of a shuffled control where the different features per gene were randomly

891 shuffled along the sequence 1000 times. Error bars represent the standard error of the

892 proportion. 
A

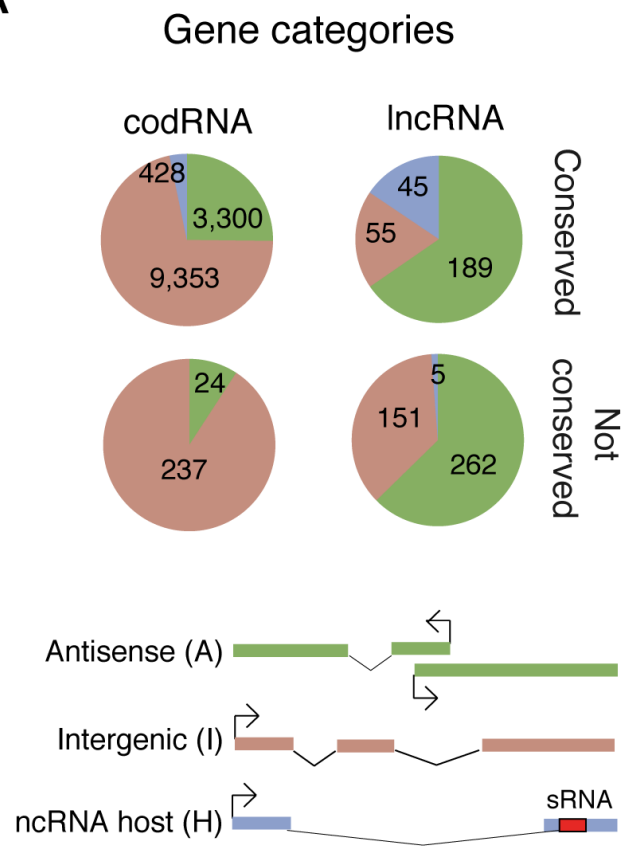

B

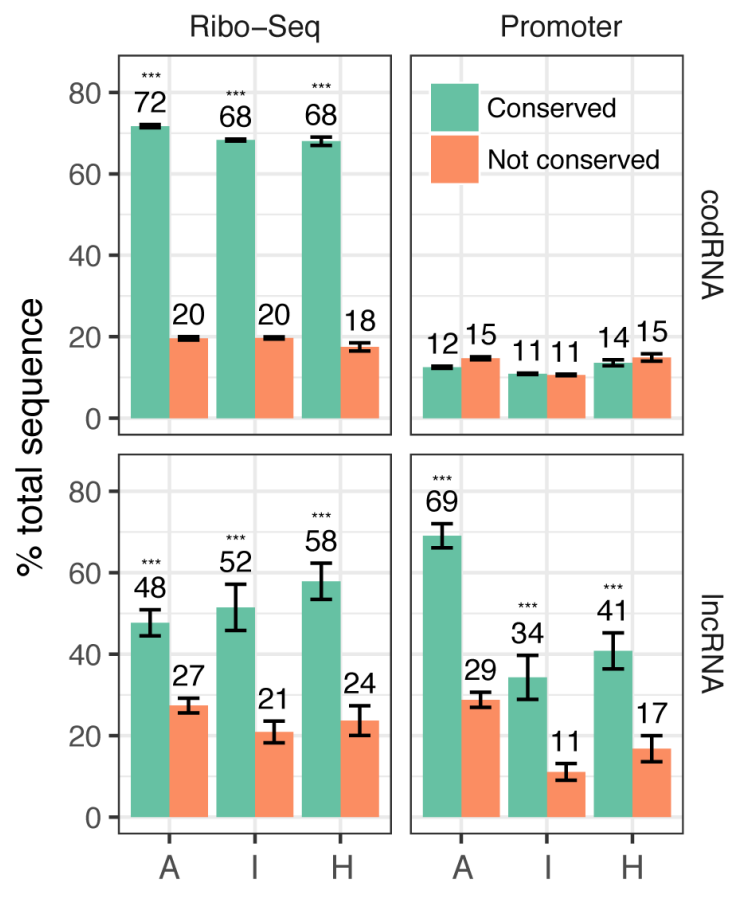

894 Figure 2. Effect of conservation across IncRNA types.

895 A. Number and fraction of different categories based on position and sequence features.

896 Antisense: Exonic overlap, expression on a bidirectional promoter, and/or annotated as

897 antisense; ncRNA host: Genes with at least one found small RNA sequence in the exonic region;

898 intergenic: rest of genes. Conserved genes are enriched in antisense and ncRNA host genes. B.

899 Percentage of total sequence that is covered by Ribo-Seq reads ( 1 or more reads), and

900 annotated promoter cores, for conserved and non-conserved regions in codRNAs and IncRNAs.

901 Conserved IncRNA regions showed a significantly higher proportion of all features compared to

902 not conserved regions or expected randomly (Test of equal proportions; * $p$-value $<0.05$; ** $p$ -

903 value $\left.<10^{-5}\right)$. Error bars represent the standard error of the proportion. Categories: A: Antisense;

904 I: Intergenic; H: ncRNA host. 


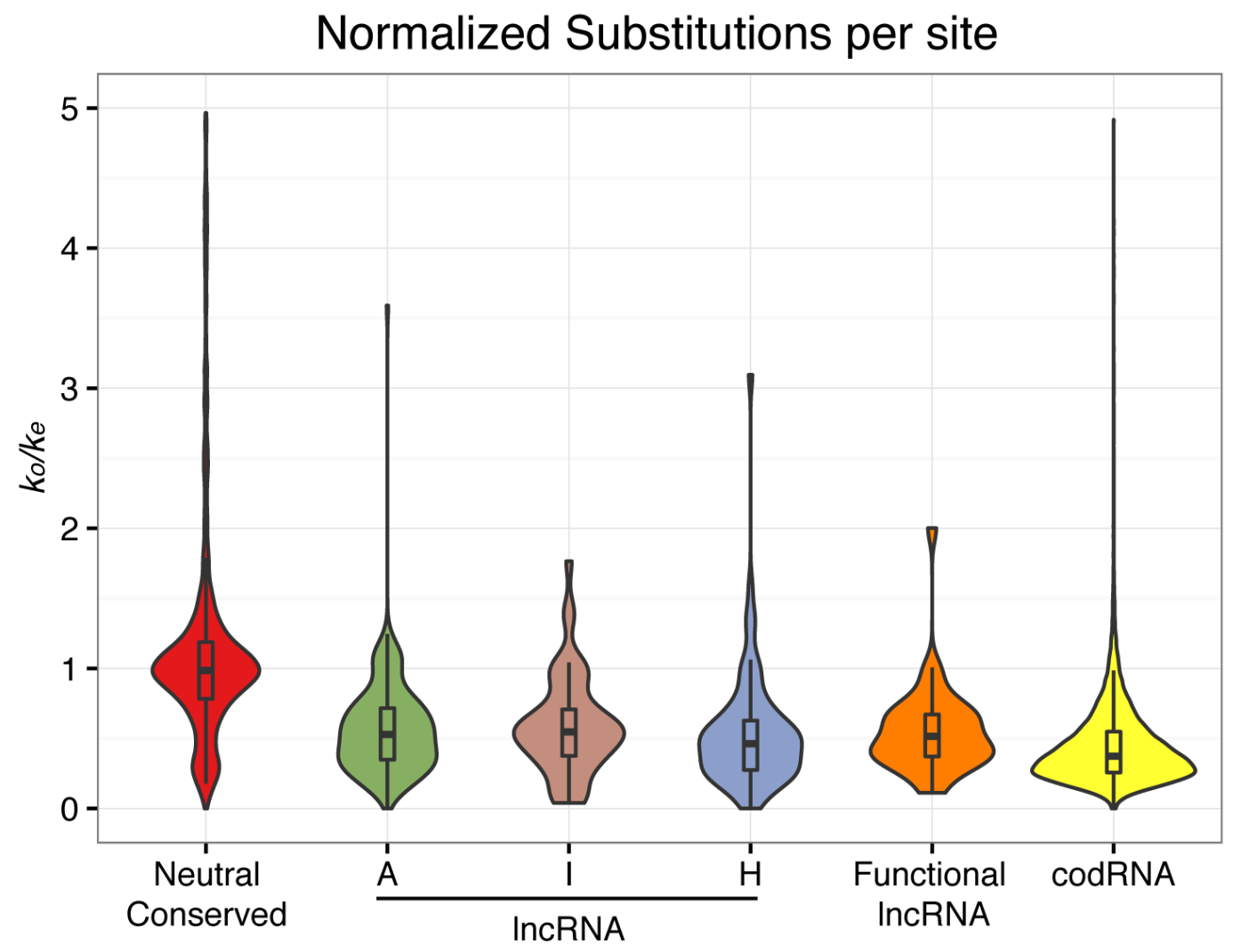

906 Figure 3. Distribution of normalized substitution rates $\left(k_{o} / k_{e}\right)$ between human and mouse

907 sequences with BLAST-based homology. The number of substitutions per site was estimated

$908\left(k_{0}\right)$ in the regions with BLAST hits with baseml under the REV nucleotide substitution model and

909 normalized by dividing it by the expected $k$ under neutrality for different length intervals $\left(k_{e}\right)$.

910 Neutral conserved: simulated neutrally evolving sequences with BLASTN matches (2,736

911 regions); IncRNA: IncRNAs with BLASTN matches; A: Antisense (179 regions); I: Intergenic (47

912 regions); H: ncRNA host (27 regions); functional IncRNA: set of 90 regions from 27 IncRNAs with

913 annotated functions in IncRNAdb; codRNA: protein coding transcripts with BLASTN matches

914 (13,034 regions). 
A

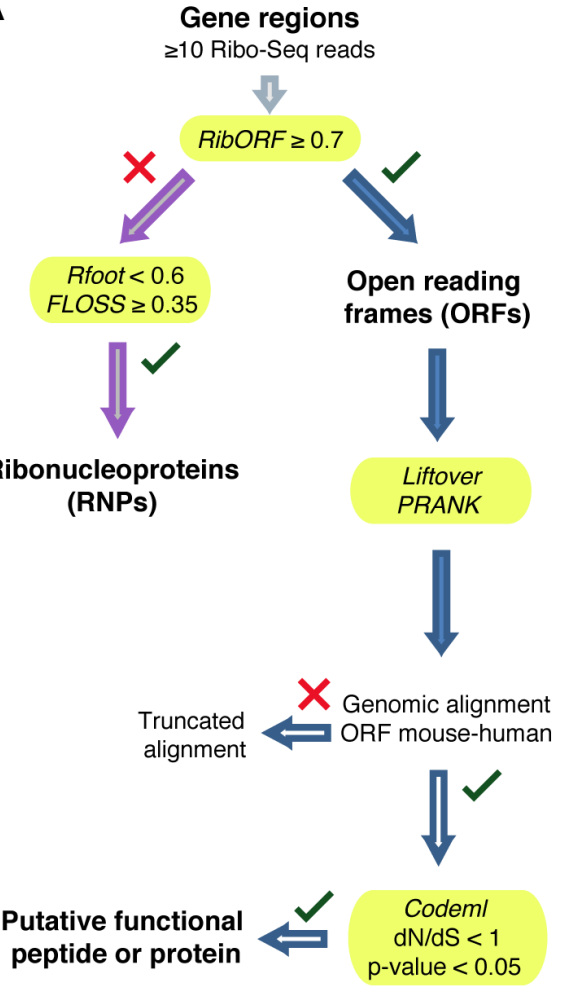

B

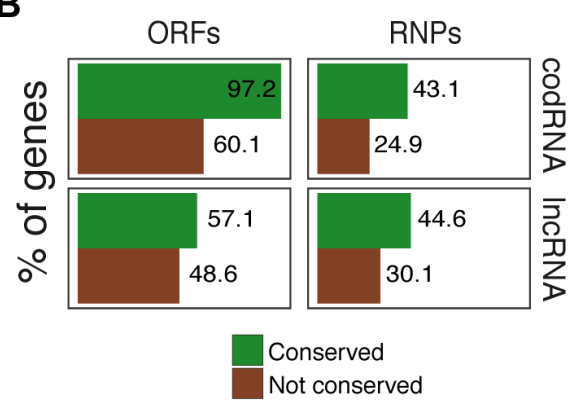

C

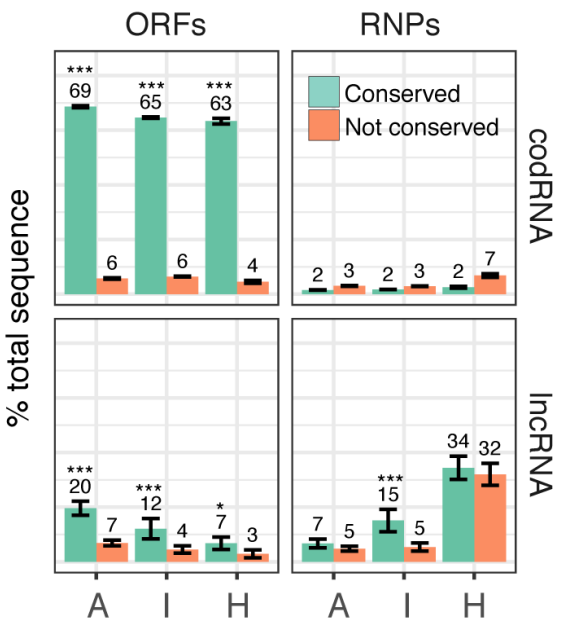

916 Figure 4. Identification of translated open reading frames and ribonucleoproteins.

917 A. Workflow to identify translated open reading frames (ORFs), putative functional proteins, and

918 ribonucleoproteins (RNPs). Ribosome profiling (Ribo-Seq) reads are mapped to candidate gene

919 regions and ORFs with a RibORF score $>=0.7$ are defined as translated. Rest of regions with

920 Rfoot uniformity score $<0.6$ and FLOSS score $>=0.35$ are defined as RNPs. Next, human ORF

921 syntenic regions are extracted with LiftOver and aligned with PRANK, when possible. Truncated

922 alignments are those ones in which less than $50 \%$ of the ORF was aligned, or the gap limit is

923 exceeded (33\% or 10-nt). Finally, non-truncated alignments are checked for purifying selection

924 signatures with Codeml to identify putative functional peptides or proteins (dN/dS ratio $<1$; Chi-

925 square test of dN/dS ratio, $p$-value $<0.05)$. B. Percentage of conserved and not conserved

926 codRNAs and IncRNAs that contain at least one translated open reading frame (ORFs) or

927 ribonucleprotein (RNPs). Conserved genes show an enrichment in ORFs and RNPs. C.

928 Percentage of total sequence that is covered by open reading frames (ORFs), and 
929 ribonucleproteins (RNPs), for conserved and non-conserved regions. CodRNA and IncRNA

930 regions showed a significantly higher proportion of ORFs compared to not conserved regions or

931 expected randomly (Test of equal proportions; * $\mathrm{p}$-value $<0.05$; ${ }^{\star \star \star}$ p-value $<10^{-5}$ ). Error bars

932 represent the standard error of the proportion. Categories: A: Antisense; I: Intergenic; H: ncRNA

933 host.

A

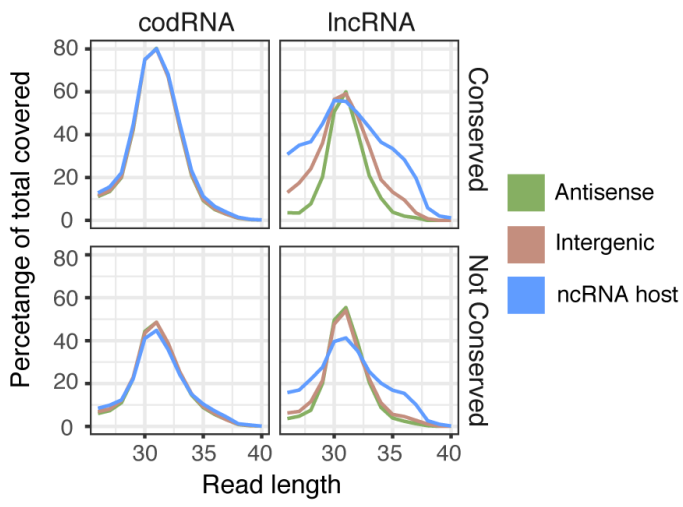

B

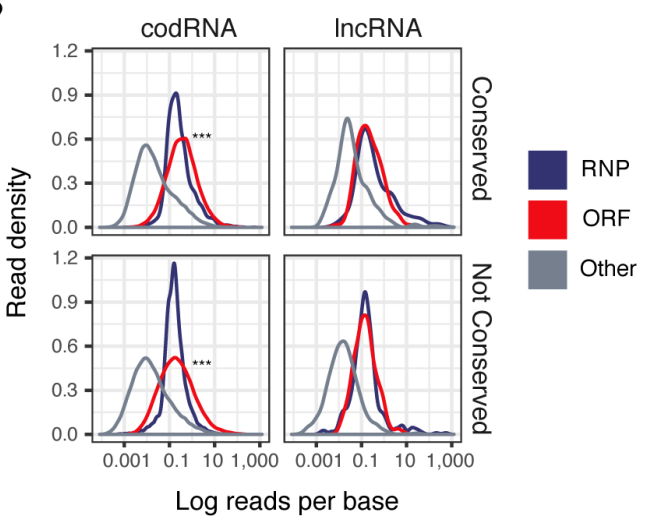

935 Figure 5. LncRNAs have more heterogenous Ribo-Seq read length.

936 A. Fraction of sequence covered by Ribo-Seq that contains reads from a specific length for

937 conserved and not conserved regions in different categories of IncRNAs. While antisense

938 IncRNAs resemble codRNAs in the read distribution, intergenic and ncRNA host regions contain

939 a higher proportion of short and long reads corresponding to non-ribosome associates. B. Ribo-

940 Seq read density for regions predicted as ribonucleoproteins (RNP), translated sequences

941 (ORF) and other regions covered by Ribo-Seq. ORFs in codRNAs have a higher read density

942 than the rest of sequences (***. Wilcoxon test, $\mathrm{p}$-value $<10^{-5}$ ) 